\title{
EA-Based Design of a Nonlinear PID Controller Using an Error Scaling Technique*
}

\author{
Gun-Baek SO \\ Division of Marine Engineering at Korea Maritime and Ocean University, \\ 727 Taejongro, Yeongdo-gu, Busan, 49112, South Korea \\ superior3608@naver.com
}

\begin{abstract}
In controller design, linear PID controllers have something of a dilemma relationship: a fast response requires large gains, which in turn, give rise to a large overshoot. For this reason, a variety of feedback control forms and related tuning methods have been implemented to guarantee satisfactory performance. Recently, multiple studies which introduce nonlinearities into the structure of the standard PID controller have been performed to solve this conflicting relationship. This study proposes an EA-based nonlinear PID controller with a first-order filter added to the derivative action to achieve the desirable quick response with low overshoot. This is realized by introducing a new type of nonlinearities in the controller gains that are time-varying functions in terms of the error and/or error rate. In addition, the nonlinear controller is designed by considering a saturation element. Then, the parameters of the nonlinear PID controller are optimally tuned by an evolutionary algorithm. In tuning the nonlinear PID controller gains, the integral of time-weighted absolute error is used as the performance evaluation of the overall control system. A set of simulation works performed on two processes with actuator saturation shows the feasibility of the proposed method.
\end{abstract}

Keywords: Nonlinear PID controller, Nonlinear functions, Saturation, Evolutionary algorithm, ITAE.

\section{Introduction}

Although many complex and delicate control techniques have been developed, proportional, integral and derivative (PID) controllers have been the most commonly used in industrial sites. The ubiquity of PID controllers enables the designing and tuning operations to be made easier in comparison with other sophisticated instruments, and enables engineers in the field to operate them relatively easily. But despite these advantages, conventional linear PID (LPID) controllers display a conflicting relationship: a fast response requires large gains, which in turn give rise to a large overshoot. As such, there is a tradeoff between fast response speed and less overshoot in the actual applications.

To solve this fundamental problem, a series of studies which introduce nonlinearities into the structure of the standard PID controller and change them online have been performed in the recent years (Jiang \& Gao, 2001; Zhang \& $\mathrm{Hu}, 2012$; Chen et al., 2012; Korkmaz, Aydoğdu

\footnotetext{
* This paper is an extended version of the paper called "GA-Based Design of a Nonlinear PID Controller and Application to a CSTR Process", published in Journal of the Korean Society of Marine Engineering (JKOSME), 2015, Vol. 39, No. 6, pages: 633-641, DOI: 10.5916/ jkosme.2015.39.6.633. In the current paper, a novel control method has been used so that each nonlinear timevarying gain can be achieved by multiplying the fixed gain and the scaled error by a nonlinear function and apply it at the controlled object with time delay.
}

\& Doğan, 2012). They can be divided roughly into two categories: Methods for nonlinearly scaling the magnitude of the input errors of the LPID controller using nonlinear functions, and methods for directly realizing three gains using nonlinear functions.

Jiang \& Gao (2001) suggested a nonlinear PID (NPID) controller that can realize the proportional, integral and derivative actions themselves of the LPID controller with the same type of nonlinear function, and applied it to the problem of braking in an anti-lock brake system vehicle. Meanwhile, some studies where controller gains were realized with nonlinear functions have been reported. Zhang and $\mathrm{Hu}$ (2012) proposed a nonlinear PID controller that directly implements three nonlinear gains using hyperbolic secant and exponential functions for the errors, and applied it to the generator excitation control system. Korkmaz, Aydoğdu \& Doğan (2012) suggested three nonlinear gains characterized by the Gaussian error function, and tuned the parameters in these gains using a genetic algorithm (GA).

All these studies have introduced nonlinear functions based on the error or the overall error rate when comparing it with the standard PID controller frame, which have taken various forms depending on the purpose of their use. However, when they are used in a controlled environment where measurement noise exists, the ideal derivative control may cause a derivative kick phenomenon. Furthermore, there is room 
for improvement because most of the proposed methods are based on complex hyperbolic secant or exponential functions and do not consider actuator saturation.

This paper presents a new class of NPID controller with three nonlinear gains and a firstorder filter added to the derivative term. The three gains of the NPID controller are realized by a simple nonlinear function based on the error or the error rate. Its parameters are tuned by an evolutionary algorithm (EA) to improve the set-point tracking performance of the overall control system which consists of the NPID controller, a saturator, and a process. In tuning the nonlinear PID controller gains, the integral of time-weighted absolute error (ITAE) criterion is used for the performance evaluation of the overall control system. The performance of the proposed method was compared to that of the existing methods through simulation on two processes to verify its effectiveness.

This paper consists of 5 sections in total, and contents of each section are as follows. Section 2 presents a brief overview of the linear PID controller. Section 3 describes the proposed NPID controller and discusses how to optimize the parameters of the NPID controller. Section 4 applies the proposed NPID controller to control the two processes and its performance is compared to the existing linear PID controller. Section 5 highlights the conclusion of this paper.

\section{Linear PID Controller}

Consider the closed-loop control system depicted in Figure 1. It consists of a PID controller $C(s)$, a controlled object $P(s)$ and a saturator.

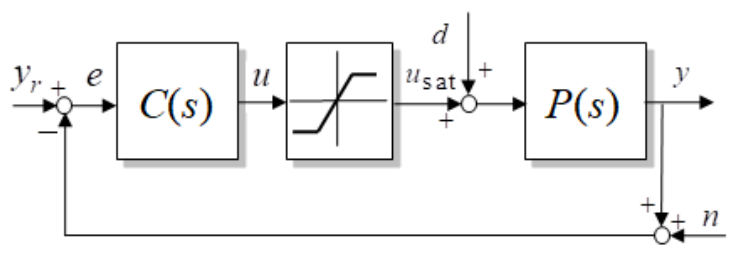

Figure 1. The PID control system

In Figure $1, y_{r}, y, d$, and $n$ denote the setpoint, measurement output, disturbance, and measurement noise, respectively; the error $e$ is defined as $e=y_{r}-y ; u$ and $u_{\text {sat }}$ are the control input and the saturator output, respectively. It is assumed that $d$ is unmeasurable.
If the standard form is used as the PID controller in Figure 1, the transfer function yields

$C(s)=k_{p}+\frac{k_{i}}{s}+k_{d} s$

where $k_{p}, k_{i}$, and $k_{d}$ denote proportional, integral and derivative gains, respectively.

The LPID controller in formula (1) consists of a parallel combination of three terms acting on the error: proportional action $\left(u_{p}\right)$, integral action $\left(u_{i}\right)$, and derivative action $\left(u_{d}\right) . u_{p}$ acts on the magnitude of the error, $u_{i}$ on the cumulative error from the initial time to the present and $u_{d}$ on the error rate. Finally the control input is calculated by adding all these values.

The performance of the closed-loop system is affected directly by the selection of $k_{p}, k_{i}$, and $k_{d} \cdot k_{p}$ contributes to accelerating system response and reduces the rise time; however, it also increases the oscillation. $k_{i}$ contributes to reducing the steady-state error but has a poor transient response; $k_{d}$ plays the role of reducing the overshoot and the settling time. As the three controls are interconnected, if one of the three gains is changed, it can be affected by the two other controls at the same time.

When the controller operates within the linear part of the saturator and the noise is disregarded, the input/output relationship of the overall control system is expressed as follows:

$$
\begin{aligned}
& Y(s)=G_{y r}(s) R(s)+G_{y d}(s) D(s) \\
& G_{y r}(s)=\frac{P(s) C(s)}{1+P(s) C(s)} \\
& G_{y d}(s)=\frac{P(s)}{1+P(s) C(s)}
\end{aligned}
$$

From formula (2), as long as the following conditions under a step change in the set-point and/or a step change in the disturbance are satisfied, the steady-state error goes to zero:

$\lim _{s \rightarrow 0} P(s) \neq 0, \lim _{s \rightarrow 0} C(s) \rightarrow \infty$

Meanwhile, the nonlinear saturator is defined by the following formula:

$u_{\text {sat }}=\left\{\begin{array}{cc}u_{\max }, & u>u_{\max } \\ u, & u_{\min } \leq u \leq u_{\max } \\ u_{\min }, & u<u_{\min }\end{array}\right.$

where $u_{\min }$ and $u_{\max }$ are the minimum and maximum values of the saturator, respectively; and $u_{\text {sat }}$ is the output of the saturator. 


\section{Proposed Nonlinear PID Controller}

As the ideal derivative term in formula (1) results in an undesirable phenomenon known as derivative kick which may occur when measurement noise is big or the set-point is abruptly changed, this study proposes an NPID controller with a firstorder filter added to the derivative term.

$C(s)=K_{p}(e)+\frac{K_{i}(e)}{s}+\frac{K_{d}(e, \dot{e}) s}{1+T_{f}(e, \dot{e}) s}$

$T_{f}(e, \dot{e})=\frac{K_{d}(e, \dot{e})}{N K_{p}(e)}$

where $K_{p}(e), K_{i}(e)$, and $K_{d}(e, \dot{e})$ are time-varying gains, which are nonlinear functions of error $e$ and/ or error rate é. $T_{f}(e, \dot{\mathrm{e}})$ is filter equation, and $N$ is a fixed value that is empirically determined between 8 and 20 (Åström \& Hägglund, 2006; O'dwyer, 2009; Rathinam, Maria \& Ramaveerapathiran, 2017). As many studies in the literature have generally used $N=10$, this value is adopted here. These functions can be expressed in various forms according to the purpose of use or the control environment. But if possible, they should be simple and allow for easy hardware implementation.

\subsection{Nonlinear Proportional Gain}

The proportional action increases in proportion to the proportional gain or the error; if too large, overshoot and oscillation may occur due to excessive control.

To increase the response speed, the proportional gain should be sufficiently large when the error is a major one, but if this big value is maintained even when the error is small after the response reaches steady-state, the effect of the error is amplified, which may cause oscillation or instability in some cases.

In this study, based on this knowledge, the magnitude of the proportional gain is adjusted in tune with that of error $e$, and the proposed $K_{p}(e)$ is a smooth function as follows:

$$
\begin{aligned}
& K_{p}(e)=k_{p} g_{p}(e) \\
& g_{p}(e)=1-\frac{1}{a_{p}+\left(c_{p} e\right)^{6}}
\end{aligned}
$$

where $k_{p}$ is a positive constant, and $g_{p}(e)$ is a nonlinear function with two user-defined parameters, $a_{p}(\geq 1)$ and $c_{p}(>0) . g_{p}(e)$ is upperbounded by 1 when $e \rightarrow \infty$ and lower-bounded by $\left(1-1 / a_{p}\right)$ when $e=0$, but its magnitude depends on the value of $a_{p}$.
Figure 2 depicts typical variations of $a_{p}$ and $c_{p}$. Meanwhile, the depth of the point where $g_{p}(e)$ becomes smaller is determined by $a_{p}$, and the smaller the $c_{p}$ value, the greater the width.

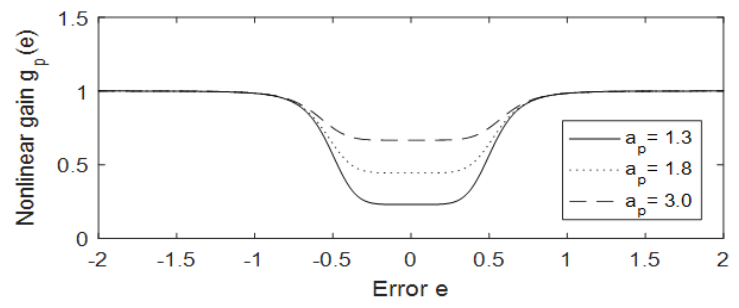

(a) $c_{p}=2.0$

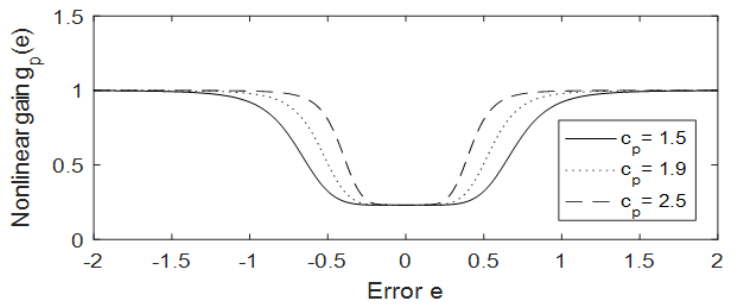

(b) $a_{p}=1.3$

Figure 2. $g_{p}(e)$ shapes to changes of $a_{p}$ and $c_{p}$

\subsection{Nonlinear Integral Gain}

The greater the absolute value of the cumulative error or the shorter the integral time, the greater the integral action. When the error is high, if the integral gain is also large, overshoot will occur; if the control input is saturated, integrator windup may occur.

Considering this fact, it is necessary to prepare for the occurrence of overshoot by reducing the integral gain value when the absolute value of error $e$ is high, and to reduce the steady-state error by increasing the integral gain value when the absolute value of error $e$ is low. For this, the following equation is used:

$K_{i}(e)=k_{i} g_{i}(e)$
$g_{i}(e)=\frac{1}{1+\left(c_{i} e\right)^{6}}$

where $k_{i}$ is the positive gain, and $g_{i}(e)$ is a nonlinear function with parameter $c_{i}(>0)$ which has a value between 0 and 1 . Figure 3 shows $g_{i}(e)$.

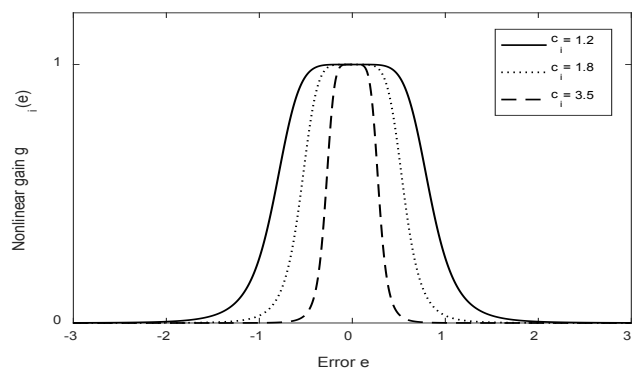

Figure 3. $g_{i}(e)$ shapes to changes of $c_{i}$ 


\subsection{Nonlinear Derivative Gain}

PID controllers are prominently featured in general process control, as it is possible to cope with high frequency noise partly by using a firstorder filter added to the derivative term. The derivative action $u_{d}$ increases in proportion to the error rate or the derivative gain and damps down by predicting in advance that if $u_{p}$ and $u_{i}$ increase, the output will increase. If damping is more than necessary during the overall control cycle, the response speed will be slow; but if damping is high during a specific cycle only, it can use $u_{p}$ and $u_{i}$ more aggressively and reduce overshoot. Thus, the size of the derivative gain is changed so that big damping can be applied when the response is in the control cycle of the red (solid) area (i.e. $e \dot{e}>0$ ), as shown in Figure 4.

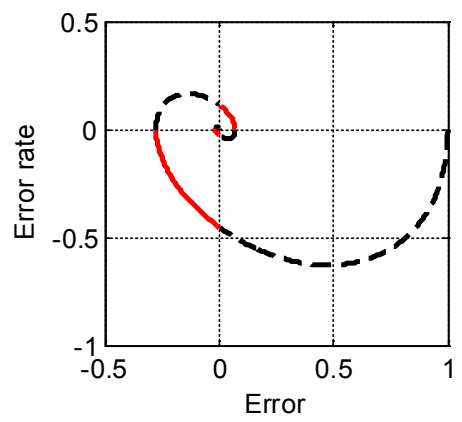

Figure 4. $\mathrm{e}-$ è phase plane

For this, the time-varying derivative gain is used as follows:

$$
\begin{aligned}
& K_{d}(e, \dot{e})=k_{d} g_{d}(e, \dot{e}) \\
& g_{d}(e, \dot{e})=\left\{\begin{array}{cc}
1-\frac{1}{a_{d}+\left(\mathrm{c}_{d} e\right)^{6}}, & e \dot{e}>0 \\
1-\frac{1}{a_{d}}, & \text { elsewhere }
\end{array}\right.
\end{aligned}
$$

where $k_{d}$ is the positive gain, and $g_{d}(e, \dot{\mathrm{e}})$ is a nonlinear function of two parameters $a_{d}(\geq 1)$ and $c_{d}(>0)$ and also has a value between 0 and 1 .

If the absolute value of error $e$ is high on the $e \dot{e}>0$ plane, $g_{d}(e, \dot{\mathrm{e}})$ will converge to 1 ; on the contrary, if the absolute value of error $e$ is low, it will converge to $\left(1-1 / a_{d}\right)$, but the size depends on the value of $a_{d}$.

\subsection{Optimal Tuning of the NPID Controller Gains}

As seen before, the proposed NPID controller has three time-varying gains $K_{p}(e), K_{i}(e)$, and $K_{d}(e, \dot{\mathrm{e}})$ and there exist a total of eight adjusting parameters $\left\{k_{p}, k_{i}, k_{d}, a_{p}, c_{p}, c_{i}, a_{d}, c_{d}\right\}$. In this study, the parameters are tuned in the overall control system including the nonlinear saturator to obtain the optimal tracking response.

Evolutionary computation (EC) has become a common approach to solving difficult, real world problems such as optimization of functions, design of neural networks and fuzzy controllers, system identification, etc. Typical examples of EC are Genetic algorithms (GAs), Evolutionary algorithms (EAs), Simulated annealing (SA), and Taboo search (Gu, Zhang \& Gao, 2009). These are able to find a global solution without any other information except the objective function.

Recently, a new class of evolutionary algorithms which utilizes both a nature-inspired operator namely, attractor - and the dynamic mutation operator was proposed by Jin \& Tran (2010). The Attractor emulating the behavior of spiral movements is implemented using a dynamic system model. The new operator in combination with the dynamic mutation is applied to a population of solution candidates to iteratively evolve these into better and better solutions. This EA operates on a hierarchical basis as follows:

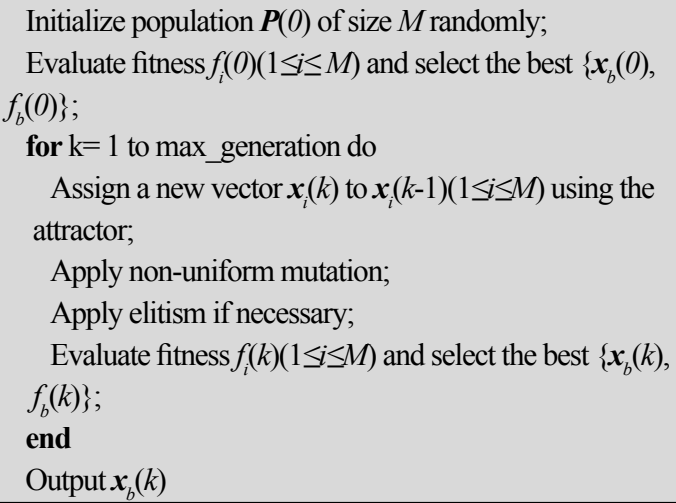

In the problem of tuning the NPID controller gains, the integral of time-weighted absolute error (ITAE) was used for the performance evaluation of the overall control system:

$J(\phi)=\int_{0}^{t_{f}} t|e(t)| d t$

where $\phi=\left[k_{p}, k_{i}, k_{d}, a_{p}, c_{p}, c_{i}, a_{d}, c_{d}\right]^{\mathrm{T}} \in R^{8}$ is a vector composed of NPID controller parameters; $e(t)$ is the error between the set-point and the output; and the integral time $t_{f}$ is large enough to render the integral after it becomes negligible. $\phi$ is tuned using an EA so that the evaluation of formula (9) is minimized.

For the EA, there have been used $P_{\text {size }}=40$ as the size of group; $\omega_{i}=0.618$ (golden ratio), $\lambda_{i}=-0.3$, 
and $m_{\max }=20$ as attractor parameters and $P_{m}=0.05$ and $b=4$ as mutation parameters.

\section{Simulation and Review}

To verify the effectiveness of the proposed NPID controller, a set of simulation works was performed through two virtual processes. For the first process, the performance of the EANPID controller was compared to that of the conventional fixed-gain PID controller tuned by the IMC method (Garcia \& Morari, 1982) and Cvejn's method (Cvejn, 2009). For the second process, the performance of the EA-NPID controller was compared with that of the NPID controller demonstrated by Korkmaz's method.

\subsection{Process I}

The first process to be controlled is an FOPTD (First order Plus Time Delay) system in formula (10) and its parameters are $\mathrm{K}=1, \tau=1[\mathrm{sec}], \mathrm{L}=$ 0.5 [sec]:

$$
P(s)=\frac{K e^{-L s}}{1+\tau s}
$$

The minimum and maximum values of the saturator were assumed to be $u_{\min }=-3$ and $u_{\max }=$ 3 , respectively.

Each parameter of the EA-NPID controller was tuned optimally in the controlled system which combines the model in formula (10) and the saturator, and the parameters were searched within the range $0<\mathrm{k}_{\mathrm{p}}, \mathrm{k}_{\mathrm{i}}, \mathrm{k}_{\mathrm{d}} \leq 10,0.1 \leq \mathrm{a}_{\mathrm{p}}, \mathrm{c}_{\mathrm{p}}$, $c_{i}, a_{d}, c_{d} \leq 10$.

Table 1 shows the summary of the parameters tuned by the proposed method and the existing methods.

Table 1. Tuned parameters for process I

\begin{tabular}{|c|c|c|c|c|c|c|c|c|}
\hline \multirow{2}{*}{ Method } & \multicolumn{7}{|c|}{ Parameters } \\
\cline { 2 - 10 } & $k_{p}$ & $k_{i}$ & $k_{d}$ & $a_{p}$ & $c_{p}$ & $c_{i}$ & $a_{d}$ & $c_{d}$ \\
\hline Proposed & 1.74 & 1.62 & 0.97 & 3.12 & 9.54 & 0.88 & 1.45 & 5.50 \\
\hline IMC & 2.78 & 2.22 & 0.56 & - & - & - & - & - \\
\hline Cvejn & 1.75 & 1.5 & 0.25 & - & - & - & - & - \\
\hline
\end{tabular}

\subsubsection{Response to Set-point (SP) Change}

To check the SP tracking performance of the proposed controller, unit step response simulation was conducted and the results were compared with those of the other methods.
As Figure 5 shows, all the controllers are able to track the step-wise variation without steady-state error, but the proposed NPID controller reaches it faster and with smaller overshoot than the other controllers. The response of the IMC method is the poorest, whereas Cvejn's method shows a long settling time.

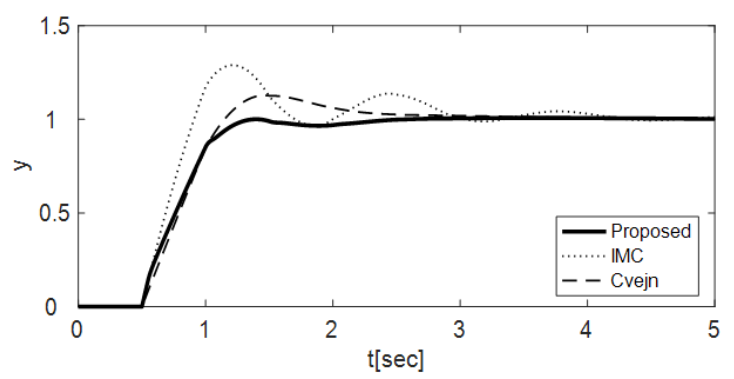

(a) Set-point tracking responses

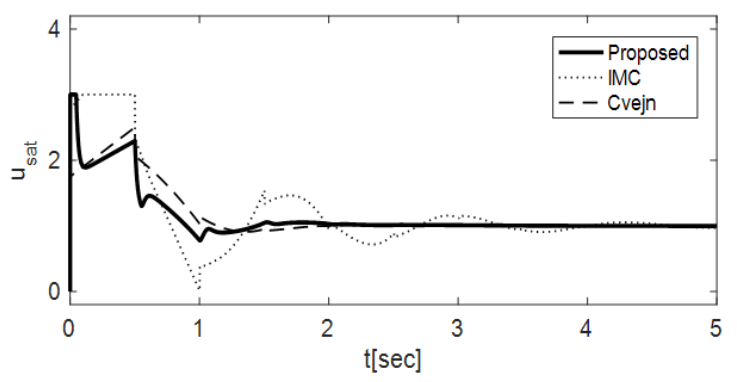

(b) Saturator outputs

Figure 5. Set-point tracking responses and saturator outputs

For the quantitative performance comparison, overshoot $M_{p}$, rise time $t_{r}\left(t_{20}-t_{10}\right), 2 \%$ settling time $t$, and the integral of the absolute error (IAE) were calculated and listed in Table 2. Here, $t_{10}$ and $t_{90}$ refer to the times required for the output to reach $10 \%$ and $90 \%$ of the set point, respectively.

It can be clearly seen that the proposed methods exhibit smaller overshoot and reduced settling time than the other methods.

Table 2. Set-point tracking performances

\begin{tabular}{|c|c|c|c|c|}
\hline \multirow{2}{*}{ Method } & \multicolumn{4}{|c|}{ Performances } \\
\cline { 2 - 5 } & $M_{p}[\%]$ & $t_{r}[\mathrm{sec}]$ & $t_{s}[\mathrm{sec}]$ & IAE \\
\hline Proposed & 0.78 & 0.54 & 2.17 & 0.32 \\
\hline IMC & 28.9 & 0.32 & 4.10 & 0.45 \\
\hline Cvejn & 12.77 & 0.49 & 2.90 & 0.42 \\
\hline
\end{tabular}

\subsubsection{Response to Noise Rejection}

A simulation was carried out to verify the performance of the proposed controller in the presence of noise. The output was perturbed by an additive white Gaussian noise component $\mathrm{N}\left(0,0.002^{2}\right)$. 
Figure 6 shows that the proposed methods have little change in the responses, but the responses of the other methods are severely distorted due to the ideal derivative action. The quantitative performance comparisons were listed in Table 3.

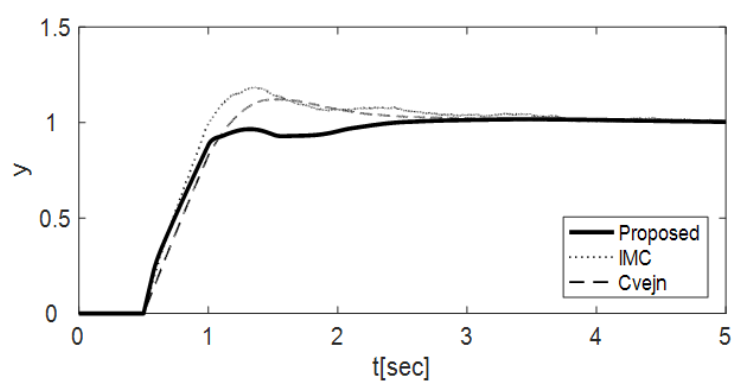

(a) Set-point tracking responses
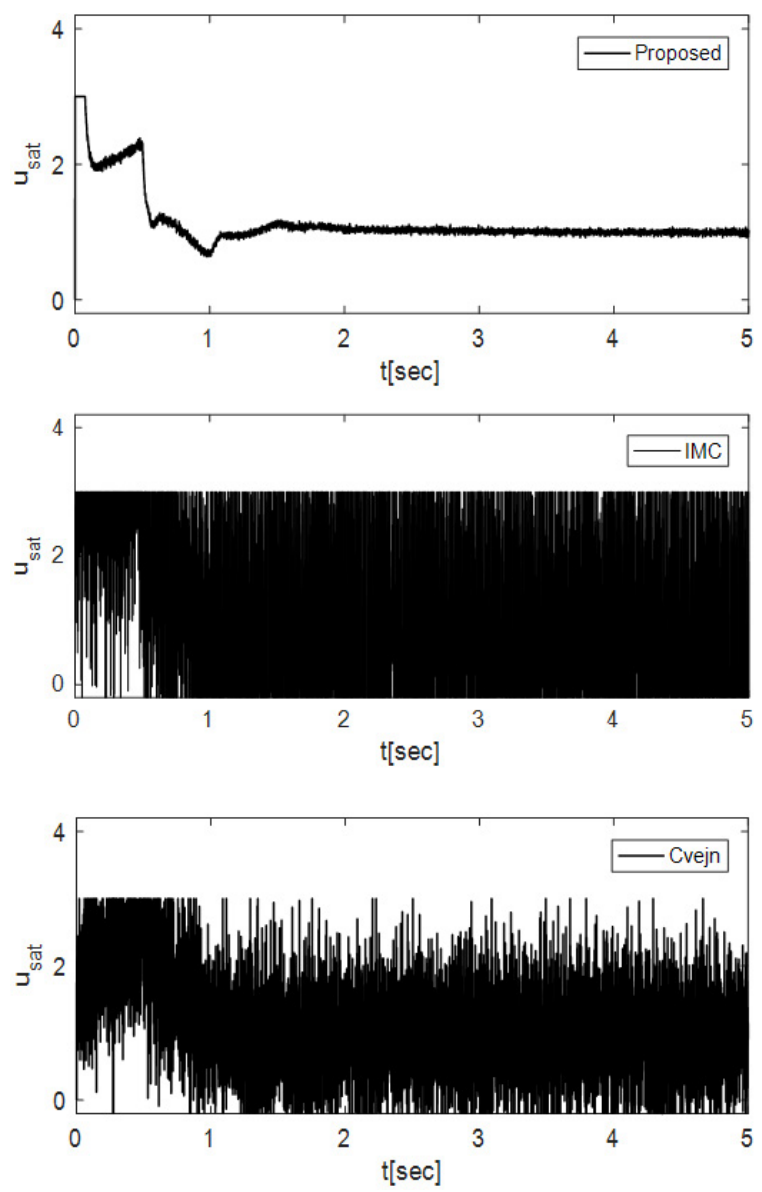

(b) Saturator outputs

Figure 6. Set-point tracking responses under Gaussian noise $\mathrm{N}\left(0,0.002^{2}\right)$

Table 3. Noise rejection performances

\begin{tabular}{|c|c|c|c|c|}
\hline \multirow{2}{*}{ Method } & \multicolumn{4}{|c|}{ Performances } \\
\cline { 2 - 5 } & $M_{p}[\%]$ & $t_{r}[\mathrm{sec}]$ & $t_{s}[\mathrm{sec}]$ & IAE \\
\hline Proposed & 1.59 & 0.48 & 2.21 & 0.34 \\
\hline IMC & 20.3 & 0.40 & 4.18 & 0.47 \\
\hline Cvejn & 12.3 & 0.50 & 3.17 & 0.44 \\
\hline
\end{tabular}

Comparing the saturator outputs in Figure 6, it can easily be observed that the severe fluctuations of $u_{\text {sat }}$ in the IMC method and Cvejn's method were greatly reduced in the EA-NPID controller response.

\subsubsection{Response to Parameter Change}

Next, the sensitivity of the system to parameter changes was verified. It was assumed that the gain $K$ and time constant $\tau$ of process I change most severely. A simulation which increases these values with $10 \%$ of the nominal value has been performed. As Figure 7 shows, the proposed method is less sensitive to the parameter changes than the other methods.

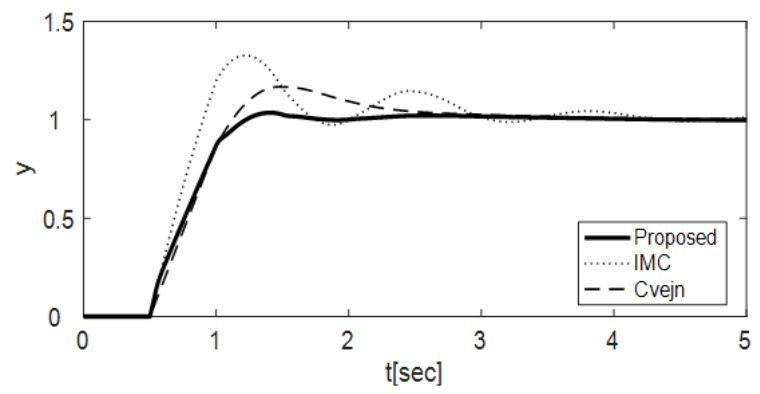

Figure 7. Set-point tracking responses to parameter changes $(K: 1 \rightarrow 1.1, \tau: 1 \rightarrow 1.1)$

The quantitative performance comparisons were listed in Table 4. It can be clearly seen that the proposed methods exhibit smaller overshoot and reduced settling time compared to the other methods.

Table 4. Parameter changing performances

\begin{tabular}{|c|c|c|c|c|}
\hline \multirow{2}{*}{ Method } & \multicolumn{4}{|c|}{ Performances } \\
\cline { 2 - 5 } & $M_{p}[\%]$ & $t_{r}[\mathrm{sec}]$ & $t_{s}[\mathrm{sec}]$ & IAE \\
\hline Proposed & 3.60 & 0.50 & 2.81 & 0.32 \\
\hline IMC & 32.77 & 0.32 & 4.14 & 0.48 \\
\hline Cvejn & 16.75 & 0.47 & 3.31 & 0.47 \\
\hline
\end{tabular}

\subsection{Process II}

\subsubsection{Response to Set-point (SP) Change}

The second process to be controlled is a thirdorder system given by Korkmaz's method and its poles are $\mathrm{p}_{1}=2, \mathrm{p}_{2}=4$.

$$
P(s)=\frac{1}{s\left(s+p_{1}\right)\left(s+p_{2}\right)}
$$

The minimum and maximum values of the saturator were assumed to be $u_{\min }=-10$ and $u_{\max }=$ 40 , respectively. 
As already mentioned, each parameter of the EA-NPID controller was optimally tuned in the controlled system which combines the model in formula (11) and the saturator, and the parameters were searched within the range $0<k_{p}, k_{i}, k_{d} \leq 100$, $1 \leq a_{p}, c_{p}, c_{i}, a_{d}, c_{d} \leq 50$.

The gains of the NPID controller proposed by Korkmaz's method are as follows:

$$
\begin{aligned}
& K_{p}(e)=a_{1}+a_{2} f(e) \\
& K_{i}(e)=b_{1}-b_{2} f(e) \\
& K_{d}(e)=c_{1}+c_{2} f(e) \\
& f(e)=\frac{2}{\sqrt{\pi}} \int_{0}^{e} \exp \left(-\tau^{2}\right) d \tau
\end{aligned}
$$

Table 5 shows the summary of the parameters tuned by the proposed method and the parameters demonstrated by Korkmaz's method.

Table 5. Tuned parameters for Process II

\begin{tabular}{|c|c|c|c|c|c|c|c|c|}
\hline Method & \multicolumn{7}{|c|}{ Parameters } \\
\hline \multirow{2}{*}{ Proposed } & $k_{p}$ & $k_{i}$ & $k_{d}$ & $a_{p}$ & $c_{p}$ & $c_{i}$ & $a_{d}$ & $c_{d}$ \\
\cline { 2 - 10 } & 55.4 & 6.23 & 32.9 & 14.0 & 17.9 & 16 & 38.6 & 7.34 \\
\hline \multirow{2}{*}{ Korkmaz } & $a_{1}$ & $a_{2}$ & $b_{1}$ & $b_{2}$ & $c_{1}$ & $c_{2}$ & - & - \\
\cline { 2 - 10 } & 28.8 & 7.2 & 6.49 & 6.49 & 7.97 & 7.97 & - & - \\
\hline
\end{tabular}

As in the previous case, Figure 8 shows the responses of the unit step input by employing the proposed method and Korkmaz's method, and Table 6 compares the quantitative performance values.

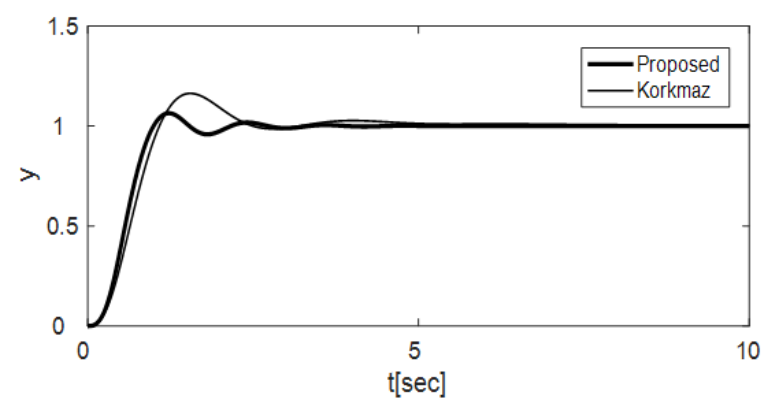

(a) Set-point tracking responses

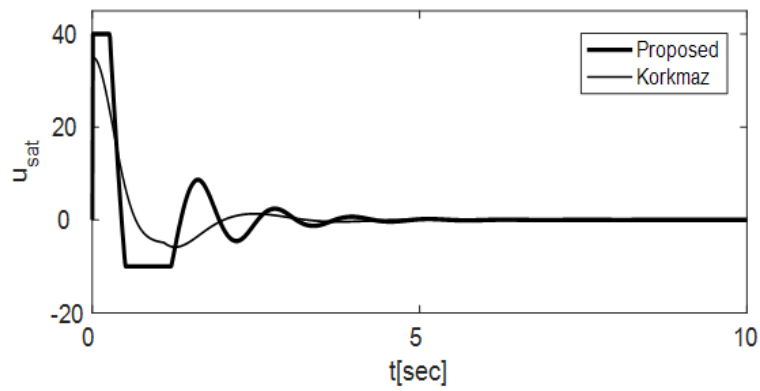

(b) Saturator outputs

Figure 8. Set-point tracking responses and saturator outputs
Table 6. Set-point tracking performances

\begin{tabular}{|c|c|c|c|c|}
\hline \multirow{2}{*}{ Method } & \multicolumn{4}{|c|}{ Performances } \\
\cline { 2 - 5 } & $M_{p}[\%]$ & $t_{r}[\mathrm{sec}]$ & $t_{s}[\mathrm{sec}]$ & IAE \\
\hline Proposed & 6.41 & 0.58 & 2.03 & 0.62 \\
\hline Korkmaz & 16.33 & 0.66 & 4.53 & 0.84 \\
\hline
\end{tabular}

As shown in the figure, both methods approach the set-point as oscillations decrease with time, but the proposed method has better performance than Korkmaz's method.

\subsubsection{Response to Noise Rejection}

As in the previous case, the output was also perturbed by an additive white Gaussian noise $\mathrm{N}\left(0,0.01^{2}\right)$.

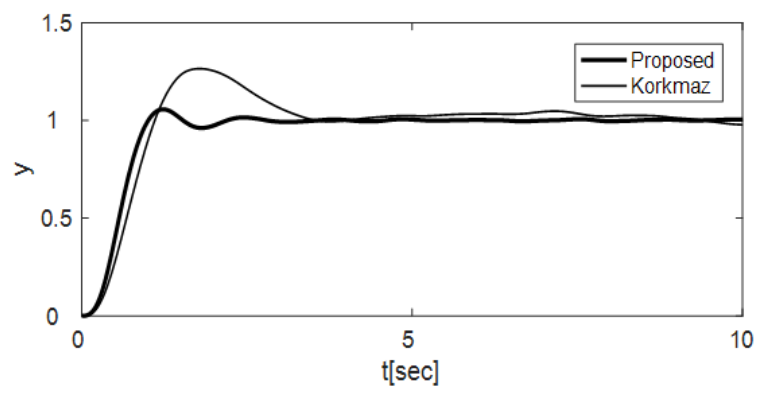

(a) Set-point tracking responses
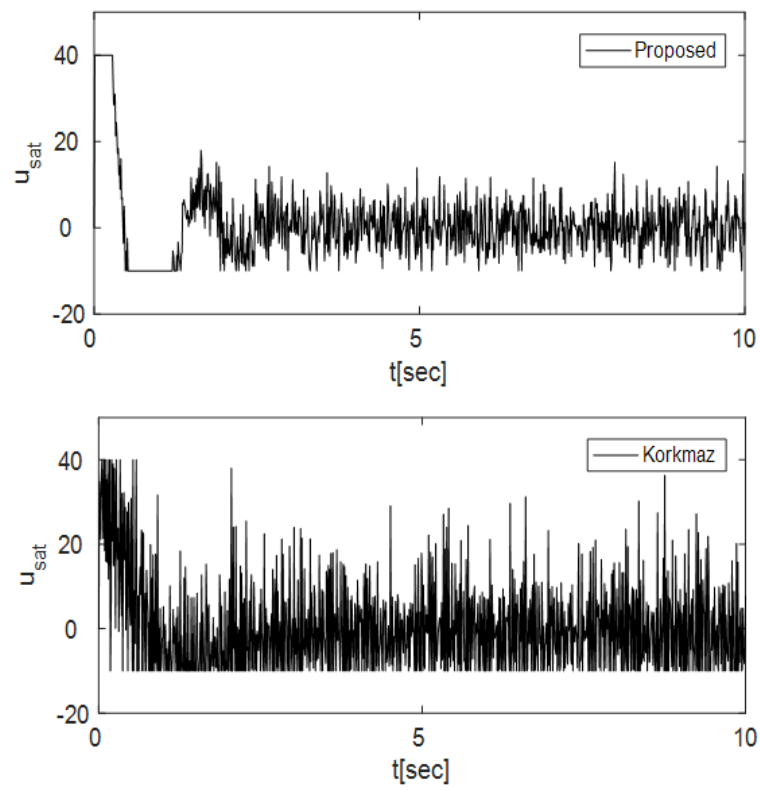

(b) Saturator outputs

Figure 9. Set-point tracking responses under Gaussian noise $\mathrm{N}\left(0,0.01^{2}\right)$

As it can be seen in Figures 8 and 9, the Korkmaz's response has been distorted, but the difference between the responses of the proposed method is hardly noticeable. Comparing the two saturator outputs in Figure 9, it can easily be noticed that 
severe fluctuations of $u_{\text {sat }}$ in Korkmaz's response have been greatly reduced in the EA-NPID controller response.

As shown in Table 7, Korkmaz's method shows poor performance due to higher overshoot as well as longer settling time.

Table 7. Noise rejection performances

\begin{tabular}{|c|c|c|c|c|}
\hline \multirow{2}{*}{ Method } & \multicolumn{4}{|c|}{ Performances } \\
\cline { 2 - 5 } & $M_{p}[\%]$ & $t_{r}[\mathrm{sec}]$ & $t_{s}[\mathrm{sec}]$ & IAE \\
\hline Proposed & 5.66 & 0.59 & 2.05 & 0.63 \\
\hline Korkmaz & 26.4 & 0.68 & - & 1.17 \\
\hline
\end{tabular}

\subsubsection{Responses to Parameter Change}

It was assumed that the pole $p_{1}$ of the open loop transfer function in formula (11) for process II changes. A simulation which increases this value with $10 \%$ of the nominal value has been performed. It is shown in Figure 10 that the proposed method is less sensitive to the parameter change than Korkmaz's method.

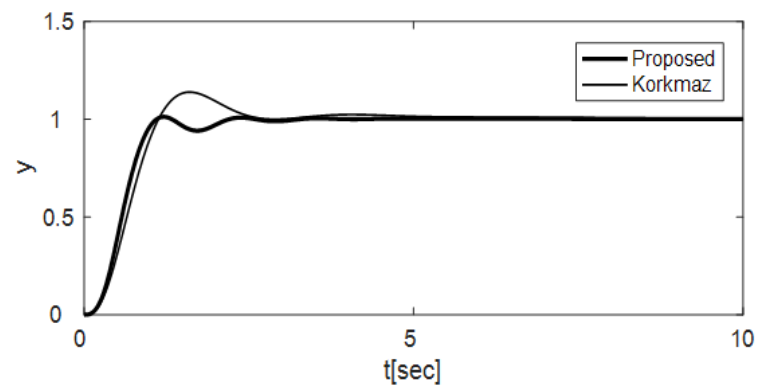

Figure 10. Set-point tracking responses to parameter change (pole $p_{1}: 2 \rightarrow 2.2$ )

The quantitative performance comparisons were listed in Table 8 . It can be clearly seen

\section{REFERENCES}

1. Åström, K. J. \& Hägglund, T. (2006). Advanced PID Control. The Instrumentation, Systems, and Automation Society.

2. Chen, J. P., Lu, B. C., Fan, F., Zhu, S. C. \& Wu, J. X. (2012). A Nonlinear PID Controller for Electro-Hydraulic Servo System Based on PSO Algorithm, Applied Mechanics and Materials, 141, 157-161.

3. Cvejn, J. (2009). Sub-optimal PID Controller Settings for FOPDT Systems that the proposed methods exhibit smaller overshoot and a reduced settling time compared to Korkmaz's method.

Table 8. Parameter changing performances

\begin{tabular}{|c|c|c|c|c|}
\hline \multirow{2}{*}{ Method } & \multicolumn{4}{|c|}{ Performances } \\
\cline { 2 - 5 } & $M_{p}[\%]$ & $t_{r}[\mathrm{sec}]$ & $t_{s}[\mathrm{sec}]$ & IAE \\
\hline Proposed & 1.28 & 0.62 & 2.05 & 0.63 \\
\hline Korkmaz & 13.9 & 0.69 & 4.57 & 0.84 \\
\hline
\end{tabular}

\section{Conclusion}

In the design of controllers, there is a Catch-22 relationship between fast response characteristics and low overshoot. A wide range of research studies has been performed in order to address this situation.

This paper presents an EA-based nonlinear PID controller to achieve the desirable quick response with low overshoot. In addition, each nonlinear time-varying gain has been achieved by multiplying the fixed gain and the scaled error by a nonlinear function. The parameters of the proposed controller have been tuned in the overall control system including the nonlinear saturator using an evolutionary algorithm. In the tuning of the parameters, the ITAE is used for the performance evaluation of the overall control system. The results of simulations performed through the two processes have confirmed that the performance of the proposed method is superior to that of other methods.

with Long Dead time, Journals of Process Control, 19(9), 1486-1495.

4. Garcia, C. E. \& Morari, M. (1982). Internal model control. A unifying review and some new results, Industrial Engineering Chemistry Process Design and Development, 21(2), 308-323.

5. Gu J. J., Zhang, Y. J. \& Gao, D. M. (2009). Application of Nonlinear PID Controller in Main Steam Temperature Control. In 
Proceedings of 2009 IEEE Conference on Power and Energy Engineering (pp. 1-5).

6. Jiang, F. \& Gao, Z. (2001). An Application of Nonlinear PID Control to a Class of Truck ABS Problems. In Proceedings of the 40th IEEE Conference on Decision and Control (pp. 516-521).

7. Jin G. G. \& Tran T. D. (2010). A NatureInspired Evolutionary Algorithm Based on Spiral Movements. In Proceeding of the SICE Annual Conference 2010 (pp.1643-1647).

8. Korkmaz, M., Aydoğdu, Ö. \& Doğan, H. (2012). Design and performance comparison of variable parameter nonlinear PID controller and genetic algorithm based PID controller. In Proceedings of the 2012 IEEE International Symposium on Innovations in Intelligent Systems and Applications (pp. 1-5).

9. O’Dwyer, A. (2009). Handbook of PI and PID Controller Tuning Rules. Imperial College Press.

10. Rathinam, M., Maria Siluvairaj, W. I. \& Ramaveerapathiran, A. (2017). Tuning of Robust PID Controller with Filter for SISO System Using Evolutionary Algorithms, Studies in Informatics and Control, 26(3), 277-286. DOI: 10.24846/v26i3y201703

11. Zhang, H. \& Hu, B. (2012). The Application of Nonlinear PID Controller in Generator Excitation System, Energy Procedia, 17(part A), 202-207. 
